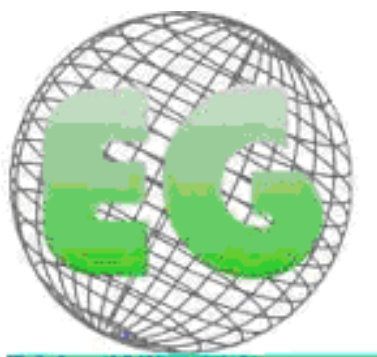

15SN $1696-6$ * *s:

$N^{\circ} 19$
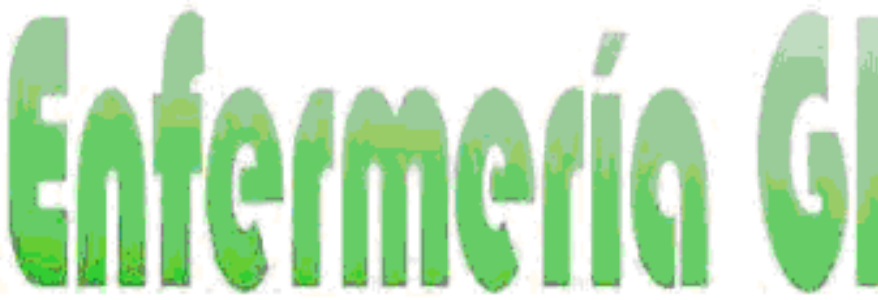

Revista electrônica cuatrimestral de Enfermeria

Junio 2010

\title{
INTERVENCIONES ENFERMERAS EN TRASTORNOS DUALES: ADICCIONES RELACIONADAS CON TRASTORNOS DE CONDUCTA EN LA INFANCIA
}

NURSING INTERVENTIONS IN DUAL DISORDERS: ADDICTION-RELATED BEHAVIOR DISORDERS IN CHILDHOOD

\begin{abstract}
*Jiménez Barbero, JA., **Rivera Rocamora, C., ***Díaz García, I., ***Pérez García, M., ${ }^{* * * * * X a n d r i ~ G r a u p e r a, ~ J M . ~}$

*Enfermero Especialista en Salud Mental. Unidad de Corta Estancia (UCE) del Hospital Psiquiátrico Román Alberca, El Palmar, (Murcia). **Profesora Titular del Departamento de Enfermería, Universidad de Murcia.

*** Enfermera Especialista en Salud Mental. Coordinadora de Rehabilitación del Centro de Salud Mental de Lorca (Murcia). ****Psiquiatra Infantil. Coordinadora de la Unidad de Infanto-Juvenil del Centro de Salud Mental de Lorca. ${ }^{* * * * *}$ Profesor Asociado Departamento de Enfermería de la Universidad de Murcia.
\end{abstract}

Palabras clave: Trastornos de conducta, trastornos por abuso de sustancias, Enfermería.

Keywords: Behavior Disorders, Substance Abuse Disorders, and Nursing.

\section{RESUMEN}

El presente estudio trata de analizar las causas y la evolución de los trastornos del comportamiento perturbador (TCP), en la infancia/adolescencia, y su repercusión en el desarrollo de un posible trastorno por abuso de sustancias (TAS), con el fin de establecer estrategias de actuación desde Enfermería, empleando para ello la taxonomía NANDA-NOC-NIC.

La metodología utilizada es la búsqueda sistemática en las principales bases de datos (Biblioteca Cochrane, Tripdatabase, Medline, Lilacs, y CUIDEN), estableciendo como criterios de selección las revisiones sistemáticas y narrativas, metaanálisis, ensayos clínicos aleatorizados (ECA'S) y no aleatorizados y estudios descriptivos u observacionales de calidad indexados en los últimos diez años. Bajo estos criterios seleccionamos finalmente un total de 20 artículos, por su especial relevancia y relación con el tema objeto del presente estudio.

En base a esta revisión, y teniendo en cuenta especialmente estudios enfermeros como el de Miotto Wright, que propone el Modelo Crítico-Holístico, esbozamos el protocolo de un Plan de Cuidados. Del análisis realizado se desprende la existencia de una relación de causa-efecto entre los TCP, cada vez más frecuentes entre niños y adolescentes y el desarrollo de un TAS, así como la gran 
influencia que en esta evolución tiene el entorno familiar y comunitario. Es por ello que consideramos que el papel de Enfermería puede ser fundamental, en los niveles de diagnóstico precoz, prevención secundaria, tratamiento y rehabilitación.

\section{ABSTRACT}

This study aims to analyze the causes and progression of Disruptive Behavior Disorders (DBD), in childhood / adolescence, and its impact on the development of a possible Substance Abuse Disorder (SAD) in order to establish action strategies from nursing, using the taxonomy NANDA-NOC-NIC.

The methodology is the systematic search of major databases (Cochrane Library, Tripdatabase, Medline, Lilacs, and CUIDEN), setting narrative and systematic reviews, meta-analysis, Randomized Clinical Trials (RCTs) and non-randomized and observational or descriptive studies indexed in the last ten years, as the selection criteria. Under these criteria finally we selected a total of 20 articles for their special relevance and relationship to the subject of this study.

Based on this review, and taking particular account of the nursing study of Miotto Wright, which proposes the Critical-Holistic Model, the protocol outlines a plan of nursing care.

The analysis reveals the existence of a cause-effect relationship between the DBD, increasingly common among children and adolescents, and the development of SAD and the great influence the family and community have on this progression. That is why, we believe that the nursing role can be crucial, in the levels of early diagnosis, secondary prevention, treatment, and rehabilitation.

\section{INTRODUCCIÓN}

Existe una estrecha relación entre los comportamientos perturbadores y el consumo de drogas en la adolescencia: por un lado, el abuso de sustancias se considera unánimemente un factor de riesgo para la realización de conductas antisociales (transgresión de normas, agresiones, acciones delictivas, etc.). Por otra parte, se ha observado que los adolescentes consumidores de alcohol y otras drogas presentan una mayor prevalencia de diagnósticos de trastorno negativista desafiante (TND), trastorno disocial (TD), y otros problemas psicopatológicos como depresión e hiperactividad. Los estudios al respecto realizados en nuestro país y en el extranjero, señalan que esta relación se da principalmente con el consumo de alcohol, pero también de marihuana, tabaco, drogas de síntesis, etc. (1).

Un modelo enfermero alternativo para el estudio de las drogas y la violencia, asociadas en muchos casos a trastornos del comportamiento perturbador (TCP), es el presentado por Miotto Wright (2000): el Modelo Crítico-Holístico de Salud Internacional ofrece una visión multidimensional de las drogas y la violencia, y una perspectiva crítico-holística de la interacción y el equilibrio entre sus componentes. El modelo parte del supuesto de que se generará una transformación en el área estudiada como resultado de una mayor conciencia de las personas que trabajan en ese sector (2).

El modelo del cuidar, característico de nuestra profesión, a pesar de haberse visto reflejado en algunos casos en el estudio de este fenómeno, carece aún de una fundamentación científica y tecnológica específica desarrollada por la profesión enfermera. La mayoría de los estudios se limitan a relatar experiencias de intervenciones por falta de una base sólida de conocimiento científico para desarrollar actividades de intervenciones a nivel de individuo, familia y comunidad (3).

El presente trabajo trata de analizar los principales estudios que relacionan los trastornos de conducta que aparecen en la infancia y la adolescencia con un posterior trastorno por abuso de sustancias (TAS), así como las interesantes posibilidades que ofrece a Enfermería para actuar, mediante la prevención, detección precoz e intervención psicofamiliar, siempre 
partiendo desde una visión holística del objeto de nuestros cuidados, en este caso, el niñoadolescente y la familia.

Para facilitar la comprensión de los términos incluimos en la tabla 1 la clasificación del Manual Diagnóstico y Estadístico de los Trastornos Mentales, Texto Revisado (DSM-IV-TR) y de la CIE-10-MC (Clasificación Internacional de Enfermedades, Modificación Clínica), para los trastornos del comportamiento perturbador.

TABLA 1: Clasificación de los trastornos del comportamiento perturbador (TCP) según DSM-IV-TR y CIE-10-MC

\begin{tabular}{|c|c|}
\hline $\begin{array}{l}\text { CIE-10: Trastornos de la conducta (TC ) y } \\
\text { Emociones }\end{array}$ & $\begin{array}{l}\text { DSM-IV: Trastorno por déficit de } \\
\text { atención y comportamiento } \\
\text { perturbador (TDA y CP) }\end{array}$ \\
\hline $\begin{array}{l}\text { F.90. Trastornos hipercinéticos: Trastornos de la } \\
\text { actividad y la atención (TAA), trastorno hipercinético } \\
\text { disocial (THD), y otros trastornos hipercinéticos. }\end{array}$ & $\begin{array}{l}\text { TDAH: combinado, inatento } \mathrm{e} \\
\text { hiperactivo-impulsivo }\end{array}$ \\
\hline $\begin{array}{l}\text { F.91 Trastornos disociales (TD) : TD en ámbito } \\
\text { familiar, TD en niños socializados y no socializados, } \\
\text { TD oposicionista desafiante (TDOD) y otros TD }\end{array}$ & $\begin{array}{l}\text { TD: -infantil } \\
\quad \text {-adolescente } \\
\quad \text {-no especificado } \\
\text { Trastorno negativista } \\
\text { (TND) } \\
\text { TCP no especificado }\end{array}$ \\
\hline $\begin{array}{l}\text { F.92 Trastornos disociales y de las emociones } \\
\text { mixtos: TD depresivo, otros TD }\end{array}$ & \\
\hline Z.62 Problemas & \\
\hline
\end{tabular}

\section{MATERIAL Y MÉTODO}

Se realizaron búsquedas en las principales bases de datos disponibles en ciencias de la salud, incluidas The Cochrane Library, Tripdatabase, Medline, Lilacs y CUIDEN, Ilevándose a cabo éstas en dos fases:

- Una primera fase en la cual se restringe la búsqueda a ensayos clínicos aleatorizados, revisiones sistemáticas y metaanálisis, publicados durante los últimos diez años: se selecciona un total de 7 estudios, que incluyen 4 revisiones sistemáticas, una de las cuales incluye un metaanálisis y 3 ensayos clínicos aleatorizados (ECA'S).

-Posteriormente, y para obtener una visión más amplia, se lleva a cabo una segunda fase, en la cual se amplían los criterios de búsqueda a ensayos clínicos no aleatorizados, estudios descriptivos y observacionales, así como revisiones narrativas y ensayos relacionados especialmente con el tema objeto del estudio.

En ambas búsquedas se emplearon los descriptores Mesh: "Conduct disorders and substance abuse disorders" que aparezcan en cualquier parte del texto del artículo. 


\section{RESULTADOS}

Finalmente se seleccionaron un total de 20 artículos, por su especial interés y relación con el tema objeto de este estudio (tabla 2).

TABLA 2: Resultados de la búsqueda bibliográfica, ordenados por orden de relevancia

\begin{tabular}{|l|l|l|l|l|l|l|}
\hline & TRIP & Cochrane & Medline & Cuiden & Lilacs & TOTAL \\
\hline $\begin{array}{l}\text { Revisiones } \\
\text { Sistemáticas }\end{array}$ & $2(4,16)$ & $1(22)$ & & & & 3 \\
\hline Metaanálisis & & & $1(21)$ & & & 1 \\
\hline ECA'S & & & $3(15,20,23)$ & & & 3 \\
\hline $\begin{array}{l}\text { Ensayos } \\
\text { Clínicos }\end{array}$ & & & $1(8)$ & & & 1 \\
\hline $\begin{array}{l}\text { Estudios } \\
\text { Observacionales }\end{array}$ & & & $1(6)$ & & & 1 \\
\hline $\begin{array}{l}\text { Estudios } \\
\text { Descriptivos }\end{array}$ & & $1(7)$ & $2(5,10)$ & $1(12)$ & & 4 \\
\hline $\begin{array}{l}\text { Revisiones } \\
\text { Narrativas }\end{array}$ & & & $4(4,9,11,13)$ & & & 4 \\
\hline Ensayos & & & & $1(1)$ & $2(2,3)$ & 3 \\
\hline
\end{tabular}

\section{Interpretación de resultados}

Realizado un análisis pormenorizado de cada uno de los estudios seleccionados, así como una lectura crítica de los mismos, se observa que las investigaciones de los últimos diez años, en relación con el tema, se han ido desarrollando en base a tres cuestiones fundamentalmente:

1) La existencia de una relación directa entre el padecimiento en la infanciaadolescencia de un trastorno de conducta, o TCP y un trastorno por abuso de sustancias (TAS), que va a persistir en la edad adulta:

Uno de los TCP que más se ha relacionado con los TAS es el trastorno por déficit de atención e hiperactividad (TDAH). De hecho la comorbilidad del TDAH y los TAS ha recibido una atención considerable en las últimas investigaciones científicas y clínicas (4-7). Estos dos trastornos están vinculados el uno al otro en una variedad de maneras. Los síntomas principales del TDAH puede ser imitado por los efectos del uso de sustancias psicoactivas, lo que dificulta el diagnóstico de un trastorno en presencia de la otra. Además, se ha demostrado que los pacientes con historia de TDAH, parecen experimentar un mayor uso indebido de drogas y con más complicaciones, lo cual indica la necesidad de iniciar estrategias de tratamiento tempranas.

La correlación TCP-TAS, se hace mucho más evidente cuando unimos a la ecuación otros trastornos de conducta como el trastorno negativista-desafiante y el trastorno disocial, tal y como evidencia un ECA reciente, realizado entre adolescentes hispanos en los que se daban ambos trastornos (8). 
2) Factores de riesgo, principalmente familiares, que van a favorecer esta asociación:

Parece haber una clara asociación entre los TCP y el TAS, pero para avanzar hacia un abordaje de naturaleza preventivo, se hace preciso conocer los factores que favorecen, en primer término, la aparición de conductas disociales en la infancia y/o adolescencia, así como los que van a predisponer a una evolución de estas hacia una toxicomanía.

Algunos autores han destacado el papel determinante de la familia en este proceso (9-11); de hecho, consideran que las causas de los TCP, pueden agruparse de acuerdo a 4 tipos de variables:

- Macroambientales: el entorno físico, socio-económico, político y cultural, compartido por un gran número de adolescentes.

- Microambientales: los contextos específicos donde se desenvuelve el adolescente, es decir, la familia, el instituto, el vecindario, la pandilla.

- Biológicas: la investigación constata respuestas psicofisiológicas anómalas al comparar adolescentes con y sin comportamientos perturbadores graves.

- Psicosociales: Los adolescentes con conductas agresivas presentan déficit en habilidades de resolución de conflictos.

Dentro de las variables mencionadas destaca la influencia del ambiente familiar, particularmente de los padres, como determinantes del ajuste psicológico y social de los hijos: la familia es el ambiente social básico del niño y el adolescente, por ser el lugar donde pasa gran parte de su tiempo. El contexto familiar puede convertirse en una fuente positiva de adaptación o, por el contrario, de estrés, dependiendo de la calidad de la relación entre los miembros de la familia (10).

En este mismo sentido, un estudio enfermero, de tipo descriptivo correlacional, publicado en 2001 (12), concluye que la intervención temprana en el desarrollo de conductas y actitudes que contemplan la interacción entre iguales y con adultos, en términos de autocontrol, respeto y resolución verbal de conflictos, protege de conductas autodestructivas y de riesgo. Asimismo protege el fortalecimiento de los vínculos familiares. Por lo tanto los factores de personalidad subyacentes a la socialización como el tipo de familia, de acuerdo al grado de cohesión que mantengan, serán factores predictores para el consumo.

3) Prevención y tratamiento, en niños y adolescentes que presentan TCP, del desarrollo de un trastorno por abuso de sustancias:

Un aspecto fundamental es sin duda la detección precoz, en edad escolar o preescolar, de aquellas conductas que pudieran ser indicativas de un posible TCP. Indudablemente, si somos capaces de detectar, en sus inicios, a aquellos niños que se encuentran en riesgo de desarrollar un trastorno de conducta, tendremos más posibilidades de evitar, no sólo que éste se materialice, sino que además culmine en un más que probable TAS. En este sentido, la Enfermera Escolar (figura que no existe aún en España), ha demostrado en otros países ser de gran importancia a la hora de detectar y prevenir los desórdenes de conducta en niños: puede ser un instrumento para educar al personal sobre los factores de riesgo y los primeros comportamientos asociados (13). 
Los distintos estudios analizados proponen varias alternativas de tratamiento en el caso de TCP asociado a TAS, ya instaurado:

-Una revisión sistemática en la que se investiga el tratamiento residencial para jóvenes con trastornos de conducta, concluye en que puede ser una intervención eficaz si está enmarcada en un sistema más amplio de atención holística, ecológica y multimodal, a la vez que destaca la gran importancia de la familia y de la terapia familiar en todo este proceso (14).

-Un ensayo clínico aleatorizado reciente, demostró la eficacia de la terapia estratégica familiar breve (BSFT), en la evolución del comportamiento de jóvenes hispanos que presentaban problemas de drogas (15).

-En contraposición, otros estudios argumentan que son imprescindibles intervenciones basadas en la comunidad, como único medio de reducir el abuso de sustancias entre jóvenes y niños especialmente con un trastorno de conducta (16).

-En el caso de TAS comórbido con TDAH, el tratamiento debería ser un acercamiento multimodal que es similar al que debería emplearse para tratar el TAS entre los pacientes que no tienen TDAH. Es esencial un acercamiento multimodal dado que el TAS, especialmente cuando es crónico y severo, responde pobremente a la mayoría de tratamientos. Además, normalmente el tratamiento del TDAH no será exitoso si no se ha estabilizado el TAS del paciente (4). Este tratamiento debería contar con educación, a paciente y familiares, sobre la enfermedad, farmacoterapia e implicaciones de ésta con el TAS. Así mismo, puede ser importante la terapia psicosocial que incluyen el entrenamiento del manejo conductual de los padres de pacientes jóvenes y la terapia cognitivo conductual (TCC) para adolescentes y adultos. Ésta será menos efectiva cuanto más avanzado y grave sea el TAS.

\section{DISCUSIÓN}

Teniendo en cuenta los conceptos que integran el metaparadigma de Enfermería (persona, salud, entorno, cuidados), así como los distintos modelos de Enfermería que se han ido proponiendo, entre los cuales destacaríamos el modelo Crítico-Holístico de Miotto Wright (2), creemos que este problema de salud abre a Enfermería un amplio campo de posibilidades de actuación. Concretamente y vistos los recursos que ofrece actualmente la red de Salud Mental en España, proponemos dos líneas de intervención enfermera, no excluyentes entre sí, sino complementarias:

A) Intervención independiente: mediante la elaboración de un plan de cuidados individualizado, siguiendo la metodología NANDA-NOC-NIC.

B) Intervención interdependiente: en colaboración con el resto del equipo multiprofesional, desde dispositivos psicoeducativos dirigidos a las familias en riesgo (grupos de padres).

\section{A) INTERVENCIÓN INDEPENDIENTE: Plan de Cuidados}

Realizaríamos, en primer lugar, una valoración, tanto del menor como de la familia, siguiendo los Patrones Funcionales propuestos por Marjory Gordon. En el caso de niños/adolescentes con un TCP y problemas de abuso de drogas o en claro riesgo de tenerlos, probablemente los patrones con más posibilidad de encontrarse alterados serían: 
-Patrón 1: percepción y manejo de la salud

-Patrón 6: cognitivo-perceptual.

-Patrón 7: autopercepción-autoconcepto.

-Patrón 8: rol-relaciones.

-Patrón 10: adaptación y tolerancia al estrés.

Por lo que respecta al diagnóstico, cabe mencionar que en un estudio reciente realizado por Plaza del Pino et al (1) entre adolescentes de Enseñanza Secundaria Obligatoria, se proponen los siguientes diagnósticos de enfermería según taxonomía NANDA:

- Mantenimiento Inefectivo de la Salud.

- Conocimientos deficientes.

- Déficit de conductas generadoras de Salud.

- Riesgo de violencia dirigida a otros.

- Riesgo de traumatismo.

Sin embargo, creemos, en base a otros estudios mencionados en un apartado anterior (911,14-16), que la prevención y el tratamiento de los TCP, desde la perspectiva enfermera, deben llevarse a cabo principalmente partiendo del seno familiar. Es en la familia donde la persona se socializa, aprende y adquiere tanto creencias, como actitudes, normas sociales interiorizadas, valores, intenciones y desarrolla a partir de ellas unas u otras conductas. El aprendizaje, la observación, las consecuencias de las acciones, etc., van poco a poco moldeando el modo de ser del niño y posteriormente del adolescente (11).

En este caso, y a modo de ejemplo, proponemos la etiqueta diagnóstica "deterioro parental" [0056], y en base a ella elaboraremos el esquema de un plan de cuidados estandarizado para familias en las que se detecte este problema.

Según NANDA-I, el "deterioro parental" se define como "incapacidad del cuidador principal para crear, mantener o recuperar un entorno favorecedor del óptimo crecimiento y desarrollo del niño" (17).

En cuanto a los factores relacionados (etiología), podrían ser en este caso:

-Temperamento difícil.

- Falta de conocimiento sobre el desarrollo del niño.

- Falta de conocimiento sobre habilidades parentales.

- Falta de habilidades de solución de problemas.

- Incapacidad para reconocer y actuar ante las claves del niño.

- Falta de habilidades de comunicación.

\section{Y las características definitorias:}

-Falta de vinculación.

-Bajo rendimiento escolar.

- Conducta inconsistente (parental).

-Cuidados incoherentes.

-Mala interacción padres-niño. 
-Verbalización de no poder controlar al niño.

- Verbalización de frustración.

Los resultados e intervenciones propuestos son:

RESULTADOS PROPUESTOS (NOC) (18):

19) Ejecución del rol de padres [2211]:

-Definición: "Acciones paternas para proporcionar un ambiente social, emocional y físico que alimente y sea constructivo para el niño".

Indicadores propuestos: de Nunca Demostrado (N/D), a Siempre Demostrado (S/D)

\begin{tabular}{|l|l|l|l|l|l|l|}
\hline Código & INDICADOR & N/D & R/D & A/D & F/D & S/D \\
\hline 221110 & $\begin{array}{l}\text { Utiliza interacciones apropiadas } \\
\text { para el temperamento del niño }\end{array}$ & 1 & 2 & 3 & 4 & 5 \\
\hline 221111 & $\begin{array}{l}\text { Utiliza control de la conducta } \\
\text { si es necesario }\end{array}$ & 1 & 2 & 3 & 4 & 5 \\
\hline 221112 & Utiliza una disciplina adecuada & 1 & 2 & 3 & 4 & 5 \\
\hline 221114 & $\begin{array}{l}\text { Interacciona de forma positiva } \\
\text { con el niño }\end{array}$ & 1 & 2 & 3 & 4 & 5 \\
\hline 221115 & Demuestra empatía hacia el niño & 1 & 2 & 3 & 4 & 5 \\
\hline
\end{tabular}

$2^{\circ}$ ) Cuidado de los hijos: seguridad psicosocial [1901]:

-Definición:" Acciones paternas para proteger a un niño de las relaciones sociales que podrían causar perjuicio o lesión".

Indicadores propuestos

\begin{tabular}{|l|l|l|l|l|l|l|}
\hline Código & INDICADOR & N/D & R/D & A/D & F/D & S/D \\
\hline 190102 & $\begin{array}{l}\text { Supervisión de los } \\
\text { Compañeros de juegos }\end{array}$ & 1 & 2 & 3 & 4 & 5 \\
\hline 190112 & $\begin{array}{l}\text { Intervención para prevenir } \\
\text { conductas sociales de riesgo }\end{array}$ & 1 & 2 & 3 & 4 & 5 \\
\hline 190117 & $\begin{array}{l}\text { Establece reglas claras de } \\
\text { conducta }\end{array}$ & 1 & 2 & 3 & 4 & 5 \\
\hline 190118 & $\begin{array}{l}\text { Mantiene una estructura y } \\
\text { una rutina diaria en la vida } \\
\text { del niño }\end{array}$ & 1 & 2 & 3 & 4 & 5 \\
\hline
\end{tabular}

3) Afrontamiento de los problemas de la familia [2600]:

-Definición: "Acciones de la familia para controlar los factores estresantes que comprometen los recursos de la familia". 
Indicadores propuestos: de Nunca Demostrado (N/D) a Siempre Demostrado (S/D)

\begin{tabular}{|l|l|l|l|l|l|l|}
\hline Código & INDICADOR & N/D & R/D & A/D & F/D & S/D \\
\hline 260001 & $\begin{array}{l}\text { Manifiesta un papel } \\
\text { de flexibilidad }\end{array}$ & 1 & 2 & 3 & 4 & 5 \\
\hline 260003 & Afronta los problemas & 1 & 2 & 3 & 4 & 5 \\
\hline 260006 & $\begin{array}{l}\text { Implica a los miembros de la } \\
\text { familia en la toma de decisiones }\end{array}$ & 1 & 2 & 3 & 4 & 5 \\
\hline 260007 & $\begin{array}{l}\text { Expresa libremente } \\
\text { sentimientos y emociones }\end{array}$ & 1 & 2 & 3 & 4 & 5 \\
\hline 260008 & $\begin{array}{l}\text { Manifiesta estrategias para } \\
\text { controlar la ira. }\end{array}$ & 1 & 2 & 3 & 4 & 5 \\
\hline 2600009 & $\begin{array}{l}\text { Utiliza estrategias para controlar } \\
\text { el estrés }\end{array}$ & 1 & 2 & 3 & 4 & 5 \\
\hline 260012 & $\begin{array}{l}\text { Establece un programa para la } \\
\text { rutina y actividades familiares }\end{array}$ & 1 & 2 & 3 & 4 & 5 \\
\hline
\end{tabular}

INTERVENCIONES PROPUESTAS (NIC) (19):

\section{Asesoramiento [5240]:}

Definición: Utilización de un proceso de ayuda interactiva centrado en las necesidades, problemas o sentimientos del paciente y sus seres queridos para fomentar o apoyar la capacidad de resolver problemas y las relaciones interpersonales.

\section{Educación paterna: crianza familiar de los niños [5566]:}

Definición: Ayuda a los padres a comprender y fomentar el crecimiento y desarrollo físico, psicológico y social de su hijo en edad preescolar o escolar.

\section{Educación paterna: adolescentes [5562]:}

Definición: Asistencia a los padres para que comprendan y ayuden a sus hijos adolescentes.

\section{Fomentar el desarrollo: niño [8274]:}

Definición: Facilitar o enseñar a los padres/cuidadores a conseguir un crecimiento global y específico lingüístico, cognitivo, social, emocional y motor total de niños en edad preescolar y escolar.

\section{Grupo de apovo [5430]:}

Definición: Uso de un ambiente grupal para proporcionar apoyo emocional e información relacionada con la salud de sus miembros. 


\section{B) INTERVENCIÓN INTERDEPENDIENTE: GRUPOS DE PADRES}

Otra oportunidad importante de abordar el problema desde Enfermería, es la colaboración con el resto del equipo multiprofesional en el desarrollo de grupos psicoeducativos dirigidos a la familia.

Diversos estudios avalan la eficacia y eficiencia de estos grupos:

Desde esta perspectiva se ha comprobado en Alemania la eficacia general del KES, un programa de formación de padres de niños con problemas de conducta, en el cual se combina una formación teórica en forma de sugerencias para el cambio, con ejercicios prácticos en formas de juegos de rol (20).

Un metaanálisis de 8 ensayos clínicos aleatorios, recomienda diversos tipos de intervención sobre la crianza de los hijos en familias con niños/as que presentan problemas de conducta, como la formación multisistémica de padres (MST) y la intervención multidimensional en hogares de guarda (MTFC) (21).

Resultan de especial interés algunos estudios que avalan, no sólo la eficacia de estos programas, sino que además parecen demostrar su eficiencia, es decir su relación costebeneficio, lo cual indica, que este tipo de intervenciones son necesarias como medio para reducir el gasto sanitario $(22 ; 23)$.

En España, este tipo de intervenciones familiares, se suelen llevar a cabo en los distintos Centros de Salud Mental Infanto-Juvenil, en los Centros de Día, asociaciones... Sin embargo, a diferencia de otros países, no existe un programa institucionalizado, ni una metodología única, aplicable en todos los Centros de la Red de Salud Mental, sino que normalmente estos grupos surgen a partir de iniciativas personales de los miembros que integran el equipo del centro, que suelen utilizar la metodología que mejor se adapta a sus recursos y posiblidades.

En cualquier, caso, estas propuestas no dejan de ser hipotéticas. No olvidemos que deberíamos siempre de tener en cuenta los factores personales, familiares, sociales y culturales que rodean al niño y a su familia. Los trastornos de conducta en la infancia y la adolescencia y su repercusión en el desarrollo de un trastorno por abuso de sustancias, constituyen hoy día, un problema difícil de detectar a tiempo, complejo de tratar y muy difícil de rehabilitar.

\section{CONCLUSIONES}

Los estudios más recientes parecen demostrar que el desarrollo de un trastorno de conducta en la infancia, además de constituir un grave obstáculo para el correcto desarrollo de la personalidad del niño/adolescente, puede ser un predictor importante de la aparición de un trastorno por abuso de sustancias.

En algunas de estas investigaciones, además se observa que en muchos de los casos en los que existe un trastorno por abuso de sustancias comórbido con un trastorno del comportamiento perturbador, se dan importantes factores de riesgo familiares, como estilo parental inadecuado, disciplina inconsistente, déficit de habilidades para la educación, etc.

La bibliografía consultada coincide en que el tipo de intervención ha de estar enmarcado en un sistema de atención holística, ecológica y multimodal, debiendo dirigirse primordialmente 
a la familia, principal fuente de socialización del niño, pero también al resto de ámbitos en que se desarrolla su personalidad: escolar, social y comunitario.

La mayoría de los investigadores, así como responsables políticos, coincide en que se necesita más investigación sobre el tratamiento precoz de este problema, que sale de lo estrictamente sanitario, e invade otras esferas como la económica, social, cultural y judicial. Este tratamiento debe no sólo reducir la disfunción actual, sino también desempeñar un papel preventivo en años posteriores.

El papel de Enfermería puede ser fundamental, en los niveles de diagnóstico precoz (Enfermera escolar), prevención secundaria, es decir una vez que se manifiestan las primeras conductas disociales en la etapa preescolar y escolar (grupos de padres, psicoeducativos, educación para la salud), tratamiento y rehabilitación (planes de cuidados individualizados, intervenciones comunitarias, tratamientos residenciales, terapia estratégica familiar breve).

Según Miotto Wright (2002), desde Enfermería es necesario desarrollar nuevos paradigmas, teorías y modelos operacionales que orienten el diseño de políticas, programas y proyectos de investigación y de intervención. La Enfermería del siglo XXI tiene que aprovechar los nuevos espacios que están abriéndose en los diferentes sectores de la sociedad. Las principales herramientas que marcarán el avance son: el liderazgo, el uso de la ciencia y tecnología, el dominio de la investigación, el desarrollo y uso del pensamiento crítico con una perspectiva holística, y el desarrollo de nuevos paradigmas y modelos teóricos.

\section{REFERENCIAS}

(1) Plaza del Pino FJ, Martínez Gálvez L, Plaza del Pino MD. Taxonomía NANDA, NIC y NOC en el Trabajo de Enfermería con Adolescentes de Educación Secundaria Obligatoria (ESO). Enfermería Docente 2007;86:31-5.

(2) MIOTTO WRIGHT M.G. A Critical-Holistic Paradigm for an Interdependent World. American Behavioral Scientist 2000;43(5):808-24.

(3) MIOTTO WRIGHT M. La contribución de la Enfermería frente al fenómeno de las drogas y la violenci en America Latina: un proceso en construcción. CIENCIA Y ENFERMERIA 2002;8(2).

(4) Faraone S.V. TDHA y trastornos por consumo de drogas. RET, Revista de Toxicomanías 2006;48:15-9.

(5) Flory K, Milich R, Lynam DR, Leukefeld C, Clayton R. Relation between childhood disruptive behavior disorders and substance use and dependence symptoms in young adulthood: individuals with symptoms of attention-deficit/hyperactivity disorder and conduct disorder are uniquely at risk. Psychol Addict Behav 2003;17(2):151-8.

(6) De Sanctis VA, Trampush JW, Harty SC, Marks DJ, Newcorn JH, Miller CJ, et al. Childhood maltreatment and conduct disorder: independent predictors of adolescent substance use disorders in youth with attention deficit/hyperactivity disorder. J Clin Child Adolesc Psychol 2008;37(4):785-93.

(7) Davids E, Von Bunau U, Specka M, Fischer B, Scherbaum N, Gastpar M. History of attention-deficit hyperactivity disorder symptoms and opioid dependence: a controlled study. Progress in Neuro-Psychopharmacology \& Biological Psychiatry 2005;29(2):291-6.

(8) Lopez B, Schwartz SJ, Prado G, Huang S, Rothe EM, Wang W, et al. Correlates of early alcohol and drug use in Hispanic adolescents: examining the role of ADHD with 
comorbid conduct disorder, family, school, and peers. J Clin Child Adolesc Psychol 2008;37(4):820-32.

(9) Espada Sánchez JP, Méndez Carrillo FX. Factores familiares, comportamientos perturbadores y drogas en la adolescencia. INTERVENCIÓN FAMILIAR EN LA PREVENCIÓN DE LAS DROGODEPENDENCIAS 2001;(1):25-55.

(10) Secades Villa R, Fernández Hermida JR. Factores de riesgo familiares para el uso de las drogas: un estudio empírico español. INTERVENCIÓN FAMILIAR EN LA PREVENCIÓN DE LAS DROGODEPENDENCIAS 2001;(2):57-112.

(11) Becoña Iglesias E. Factores de riesgo y protección familiar para el uso de drogas. INTERVENCIÓN FAMILIAR EN LA PREVENCIÓN DE LAS DROGODEPENDENCIAS 2001;(3):113-40.

(12) Ramón Florenzano U, Patricia Sotomayor C, Magdalena Otava T. Estudio comparativo del rol de la socialización familiar y factores de personalidad en las farmacodependencias juveniles. Rev chil pediatr 2001;72(3).

(13) Barcalow K. Oppositional defiant disorder: information for school nurses. J Sch Nurs 2006;22(1):9-16.

(14) Hair H J. Outcomes for children and adolescents after residential treatment: a review of research from 1993 to 2003. Database of Abstracts of Reviews of Effects (DARE) 2005.

(15) Santisteban DA, Coatsworth JD, P-VA, Kurtines WM, SS, LaPerriere A, et al. Efficacy of brief strategic family therapy in modifying Hispanic adolescent behavior problems and substance use. J Fam Psychol 2003;17(1):121-33.

(16) Adams C, Atkinson S, Bury M, Capewell S. Community-based interventions to reduce substance misuse among vulnerable and disadvantaged children and young people. NICE public health intervention guidance 2007;4.

(17) Heather Herdman T, Heath C, Meyer G. NANDA I: Diagnósticos enfermeros: definiciones y clasificación. Elsevier; 2007.

(18) Moorhead S, Johnson M, Maas M. Clasificación de resultados de Enfermería (NOC). $3^{\mathrm{a}}$ ed. Elsevier; 2005.

(19) McCloskey Dochterman J, Bulechek G. Clasificación de Intervenciones de Enfermería (NIC). 4 ${ }^{\mathrm{a}}$ ed. Elsevier; 2004.

(20) Grimm K, Mackowiak K. KES--training for parents of children with conduct behaviour problems. Prax Kinderpsychol Kinderpsychiatr 2006;55(5):363-83.

(21) Woolfenden SR, Williams K, Peat JK. Family and parenting interventions for conduct disorder and delinquency: a meta-analysis of randomised controlled trials. Arch Dis Child 2002;86:251-6.

(22) Dretzke J, Drew E, Davenport C, Barlow J, Stewart-Brown S, Sandercock J, et al. Effectiveness and cost effectiveness of parent training/ education programmes for the treatment of conduct disorder, including oppositional defiant disorder, in children. Health Technology assesment 2005;9(50).

(23) Edwards RT, Céilleachair A, Bywater T, Hughes DA, Hutchings J. Parenting programme for parents of children at risk of developing conduct disorder: cost effectiveness analysis. BMJ 2007;334(682):1-6. 